\title{
Plasma Kynurenine Predicts Severity and Complications of Heart Failure and Associates with Established Biochemical and Clinical Markers of Disease
}

\author{
Thomas Bernd Dschietzig $^{a, b} \quad$ Karl-Heinz Kellner $^{c} \quad$ Katrin Sasse $^{b} \quad$ Felix Boschann $^{b}$ \\ Robert Klüsener $^{b} \quad$ Jana Ruppert ${ }^{a} \quad$ Franz Paul Armbruster ${ }^{a} \quad$ Dragic Bankovic $^{d}$ \\ Andreas Meinitzer ${ }^{e}$ Veselin Mitrovic ${ }^{f}$ Christoph Melzer ${ }^{b}$ \\ ${ }^{a}$ Immundiagnostik AG, Bensheim, Germany; ${ }^{b}$ Department of Cardiology and Angiology, \\ Campus Mitte, Charité University Medicine Berlin, Berlin, Germany; ${ }^{\circ}$ Neuroimmun GmbH, \\ Karlsruhe, Germany; ${ }^{\mathrm{d} D e p a r t m e n t}$ of Mathematical Sciences, State University of Novi Pazar,

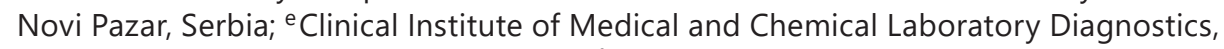

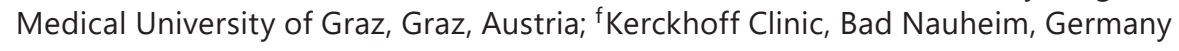

\section{Keywords}

Heart failure $\cdot$ Biomarker $\cdot$ Mortality $\cdot$ Exercise $\cdot$ Tryptophan

\begin{abstract}
Background: Kynurenine, a metabolite of the L-tryptophan pathway, plays a pivotal role in neuro-inflammation, cancer immunology, and cardiovascular inflammation, and has been shown to predict cardiovascular events. Objectives: It was our objective to increase the body of data regarding the value of kynurenine as a biomarker in chronic heart failure (CHF). Methods: We investigated the predictive value of plasma kynurenine in a CHF cohort (CHF, $n=$ 114); in a second cohort of defibrillator carriers with CHF (AICD, $n=156$ ), we determined clinical and biochemical determinants of the marker which was measured by enzyme immunoassay. Results: In the CHF cohort, both kynurenine and NT-proBNP increased with NYHA class. Univariate binary logistic regression showed kynurenine to predict death within a 6-month follow-up (OR 1.43, 95\% Cl 1.03-2.00, $p=0.033$ ) whereas NT-proBNP did not contribute significantly. Kynurenine, like NT-proBNP, was able to discriminate at a $30 \%$ threshold of left ventricular ejection fraction (LVEF; AUC-ROC, both 0.74). Kynurenine correlated inversely with $\operatorname{LVEF}(\varrho=-0.394)$, glomerular filtration fraction (GFR; $e=-0.615)$, and peak $\mathrm{VO}_{2}$ $(\varrho=-0.626)$. Moreover, there was a strong correlation of kynurenine with NT-proBNP $(e=$ $0.615)$. In the AICD cohort, multiple linear regression analysis demonstrated highly significant associations of kynurenine with GFR, hsCRP, and tryptophan, as well as a significant impact
\end{abstract}


of age. Conclusions: This work speaks in favor of kynurenine being a new and valuable biomarker of $\mathrm{CHF}$, with particular attention placed on its ability to predict mortality and reflect exercise capacity.

\section{Introduction}

Kynurenine (Kyn) is a metabolite of L-tryptophan produced by the enzymes indoleamine 2,3-dioxygenase (IDO-1 and -2) [1] and tryptophan 2,3-dioxygenase [2]. The key enzyme, IDO-1, is prevailingly activated by lipopolysaccharide, tumor necrosis factor- $\alpha$, interleukin-1, and interferon- $\gamma[1,3,4]$. Apart from exerting effects on neuro-regulation [5] and cancer immunology [6], Kyn and its metabolites have a role in vascular inflammation [7], endothelium-dependent vasodilation [8], and oxidative stress [9]. Kyn or the Kyn/tryptophan ratio have recently been suggested to predict cardiovascular prognosis, particularly in coronary artery disease (CAD) [10,11] and after cardiac arrest [12].

Data on chronic heart failure (CHF) and Kyn, however, are scarce. We have recently co-authored a report demonstrating plasma Kyn to be significantly elevated in patients with stable CHF, enrolled in the SICA-HF study, as compared to control individuals [13]. That report also showed inverse associations of circulating Kyn with measures of functional capacity, such as handgrip strength, peak oxygen consumption, and 6-min walk distance.

In the present work, we aimed at gathering more details on Kyn's performance as a CHF biomarker. In our first cohort of CHF patients, we investigated Kyn's ability to predict death in $\mathrm{CHF}$ as well as its association with parameters of CHF severity, and we compared its performance with that of the established CHF biomarker, NT-proBNP. In a second CHF cohort of AICD carriers, we evaluated several clinical and biochemical parameters as determinants of plasma Kyn using multiple linear regression analysis. Overall, the findings corroborate the upcoming notion of Kyn predicting CHF severity and complications as well as reflecting exercise capacity. Kyn furthermore associates with markers of inflammation and renal function.

\section{Materials and Methods}

\section{Patients}

All patients in the cohorts mentioned below provided informed written consent to have data from their medical records used in research. All data were fully anonymized before being accessed by researchers.

\section{CHF Cohort}

This study was approved by the Ethics Committee of the Justus Liebig University of Giessen (Kerckhoff Clinic Bad Nauheim; BioData Bank 144/11) and has been performed in accordance with the ethical standards laid down in the 1964 Declaration of Helsinki and its later amendments. The study enrolled 114 patients (mean age $71 \pm 12$ years, $72 \%$ male) with varying degrees of $\mathrm{CHF}$, either heart failure with preserved or with reduced ejection fraction: NYHA I, 6\%; NYHA II, 60\%; NYHA III, 31\%, and NYHA IV, 3\%. Peripheral venous blood was drawn at baseline for the determination of the different biomarkers (see below), and routine laboratory and clinical parameters were recorded.

Owing to logistical limitations, time-to-event data were not available in this cohort. The patients were followed up for 6 months. 
Table 1. Clinical and biochemical characteristics in both study cohorts

\section{AICD Cohort}

This study was approved by the Ethics Committee of the Charité University Medicine Berlin (approval No. EA1/004/13). The study enrolled 156 patients (mean age $68 \pm 11$ years, $17 \%$ female) from an out-patient department carrying AICD for primary or secondary prevention of sudden cardiac death. All of them suffered from CHF (33 [20\%] from heart failure with preserved ejection fraction), 71\% from CAD, and 27\% from type-2 diabetes. These patients routinely presented for device control every 6 months. Peripheral venous blood was drawn for the determination of several laboratory parameters (see below), and the routine laboratory and clinical parameters were recorded. In this cohort, neither current NT-proBNP levels nor NYHA classification were available owing to logistic limitations. The following clinical and routine biochemical parameters were available: creatinine, severity of CAD (1-, 2-, or 3-vessel disease), and left ventricular ejection fraction (LVEF).

\section{Determination of Kyn and Other Parameters}

EDTA plasma was stored at $-70^{\circ} \mathrm{C}$ for subsequent determinations. Plasma Kyn was measured using a competitive enzyme immunoassay (No. K7728, Immundiagnostik AG, Bensheim, Germany). Nitrotyrosine, zonulin, high-sensitivity C-reactive protein (hsCRP), carbonylated proteins, myeloperoxidase, tryptophan, carboxy-methyl-lysine calprotectin, 
Fig. 1. NT-proBNP and Kyn in the CHF cohort. Circulating NT-proBNP (upper plot) and Kyn (lower plot) in dependence of NYHA functional class, grouped into NYHA I-II versus NYHA III-IV (NYHA I, $n=7$; NYHA II, $n=69$; NYHA III, $n=35$; NYHA IV, $n=3$ ). Group comparison was done using the Mann-Whitney $U$ test. Data are shown as box plots: boxes indicate the interquartile range, the inner line being the median; whiskers indicate 1.5-fold of the interquartile range; values above 1.5 times (outlier) and 3 times the interquartile range (extreme values) are depicted as circles and stars, respectively.

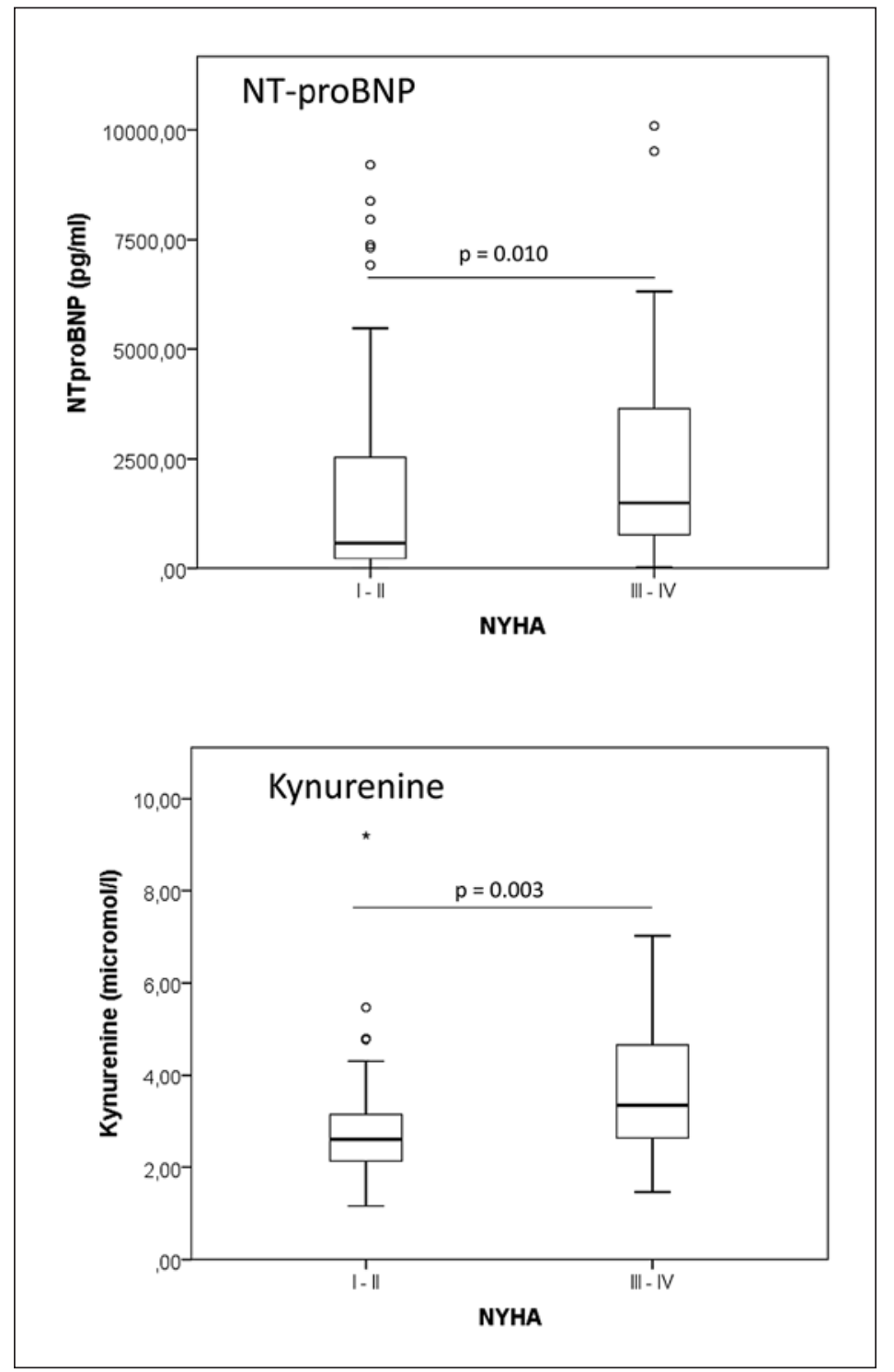

and oxidized LDL were determined using test kits from Immundiagnostik according to the manufacturer's instructions. The NT-proBNP ELISA was from Roche.

\section{Statistical Analysis}

Data are given as the median (interquartile range) unless stated otherwise. First, normality of distribution was tested using the Kolmogorov-Smirnov test. In the CHF cohort, discriminatory power was assessed using ROC analysis; combined ROC curves were produced by multivariate binary logistic regression (with stepwise backward regression). The DeLong test was used to compare individual ROC curves. To obtain a final model for death prediction, we included the significant univariate candidates into a multivariate binary logistic regression analysis.

In the AICD cohort, laboratory and clinical parameters showing a significant univariate correlation with Kyn according to Spearman's rho (@) were selected. Those parameters were then included in a multiple linear regression analysis (generalized linear model). Categorical 


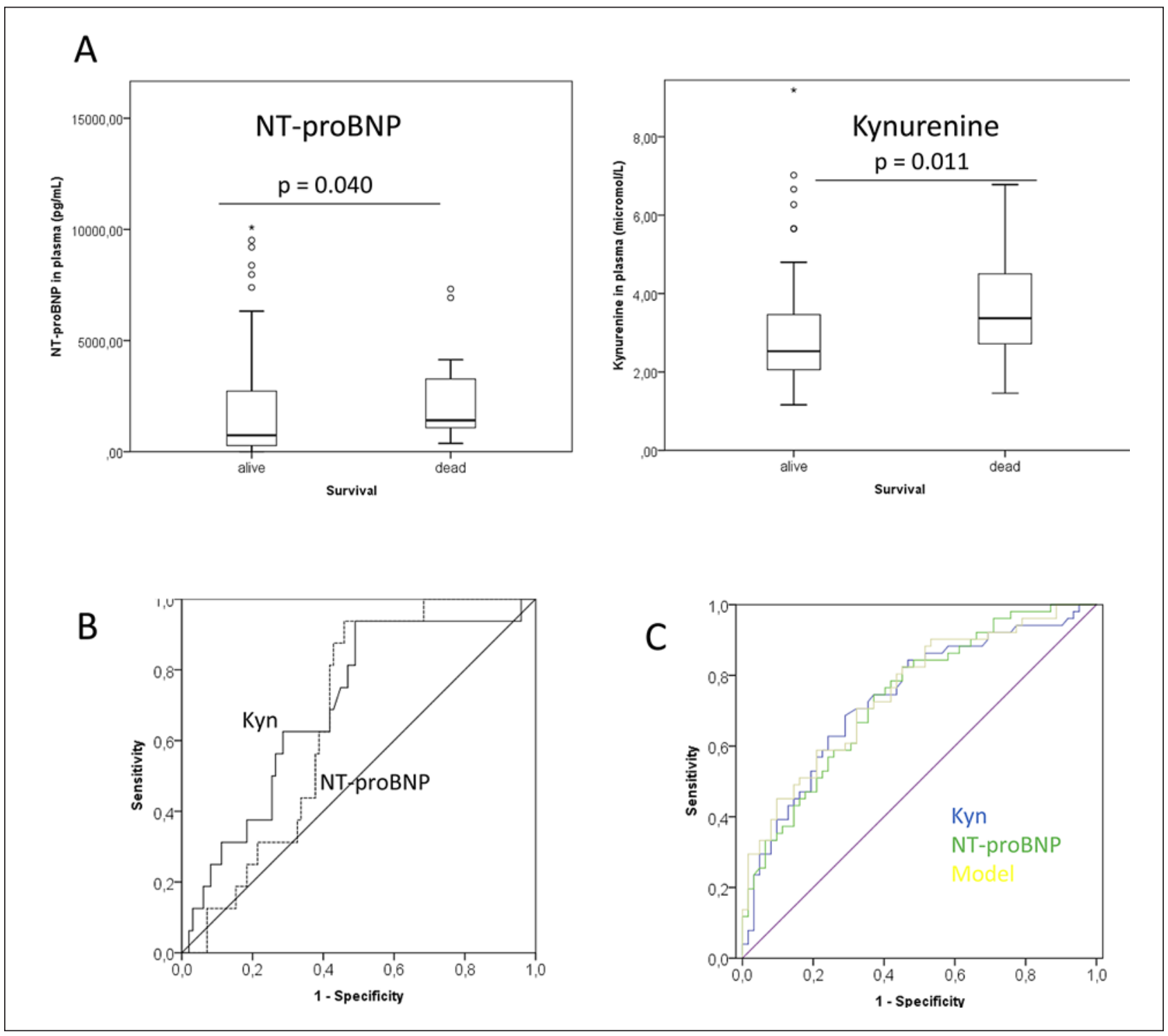

Fig. 2. A NT-proBNP and Kyn as death predictors in the CHF cohort. Circulating NT-proBNP (left) and Kyn (right) in survivors $(n=98)$ versus non-survivors $(n=16)$. Data are shown as box plots: boxes indicate the interquartile range, the inner line being the median; whiskers indicate 1.5-fold of the interquartile range; values above 1.5 times (outlier) and 3 times the interquartile range (extreme values) are depicted as circles and stars, respectively. B ROC analysis for death in the CHF cohort. ROC curves for death prediction by Kyn (solid line, AUC 0.70) versus NT-proBNP (dotted line, AUC 0.63). C ROC analysis for low LVEF in the CHF cohort. ROC curves for prediction of LVEF $<30 \%$ by Kyn (blue line), NT-proBNP (green line), and a combination thereof (yellow line). The combined ROC curve was constructed by multivariate binary logistic regression: Kyn, OR 1.57 (95\% CI 1.05-2.35); NT-proBNP, OR 1.029 (95\% CI 1.003-1.055).

variables were included in the model if the Mann-Whitney U test indicated significant differences of Kyn between categories.

In general, differences between groups were analyzed using the non-parametric MannWhitney $\mathrm{U}$ test. $p<0.05$ was regarded as significant and $p<0.005$ as highly significant. All analyses were carried out with IBM SPSS Statistics 23. 
Kidney

Blood Pressure

Research

Table 2. Death prediction in the CHF cohort - logistic regression analysis

\begin{tabular}{l|l}
\hline Kidney Blood Press Res 2019;44:765-776 \\
\hline DOI: 10.1159/000501483 & $\begin{array}{l}\text { @ 2019 The Author(s). Published by S. Karger AG, Basel } \\
\text { www.karger.com/kbr }\end{array}$ \\
\hline
\end{tabular}

\begin{tabular}{|c|c|c|c|c|}
\hline & Coefficient B & OR & $95 \%$ CI & $p$ value \\
\hline \multicolumn{5}{|c|}{ Univariate analysis } \\
\hline Kyn & 0.36 & 1.43 & $1.03-2.00$ & 0.033 \\
\hline Height & -0.08 & 0.93 & $0.87-0.99$ & 0.026 \\
\hline LVEF & -0.05 & 0.95 & $0.90-0.997$ & 0.037 \\
\hline LVEDD & 0.06 & 1.07 & $1.001-1.14$ & 0.047 \\
\hline Plasma sodium & -0.22 & 0.80 & $0.69-0.93$ & 0.005 \\
\hline CAD & 1.22 & 3.38 & $1.09-10.5$ & 0.035 \\
\hline NT-proBNP & 0.001 & 1.001 & $0.99-1.01$ & 0.90 \\
\hline \multicolumn{5}{|c|}{ Multivariate analysis - model 1} \\
\hline Kyn & 0.40 & 1.49 & $1.05-2.10$ & 0.025 \\
\hline Height & -0.08 & 0.92 & $0.86-0.99$ & 0.021 \\
\hline \multicolumn{5}{|c|}{ Multivariate analysis - model 2} \\
\hline Kyn & 0.32 & 1.37 & $0.95-2.00$ & 0.09 \\
\hline Height & -0.09 & 0.91 & $0.85-0.98$ & 0.015 \\
\hline CAD & 1.20 & 3.30 & $0.96-11.3$ & 0.06 \\
\hline \multicolumn{5}{|c|}{ Multivariate analysis - final model 3} \\
\hline Height & -0.08 & 0.93 & $0.86-0.99$ & 0.048 \\
\hline LVEDD & 0.08 & 1.08 & $1.004-1.16$ & 0.039 \\
\hline Sodium & -0.21 & 0.81 & $0.69-0.95$ & 0.011 \\
\hline
\end{tabular}

The following parameters were also tested in univariate analysis, but found to be non-significant: age, sex, weight, body mass index, heart rate at rest and during peak exercise, systolic and diastolic blood pressure, right ventricular end-diastolic diameter, left ventricular end-systolic diameter, history of myocardial infarction, NYHA class; presence of: sinus rhythm, pacemaker or defibrillator, diabetes, hypertension, atrial fibrillation, hyperuricemia; plasma/serum parameters: creatinine, urea, hemoglobin, potassium, C-reactive protein, nitrotyrosine, zonulin, tryptophan, oxidized LDL, glomerular filtration rate (MDRD formula and cystatin C-based formula). For multivariate analyses, the following models were constructed: Model 1: kyn, height. Model 2: kyn, height, CAD. Model 3: kyn, height, CAD, LVEF, LVEDD, sodium; for this model, only the final significant contributors after backward regression (Wald procedure) are shown. CI, confidence interval; LVEF, left ventricular ejection fraction; LVEDD, left ventricular end-diastolic diameter; Kyn, kynurenine. Odds ratios are given per unit increase for continuous variables.

\section{Results}

Table 1 summarizes the clinical and biochemical characteristics of the patients in the CHF and AICD cohorts.

\section{CHF Cohort}

Baseline Kyn Increases with NYHA Class and Is Predictive of Death

Figure 1 shows the significant increase of both NT-proBNP and Kyn with NYHA functional class. As there were only 3 patients in NYHA IV, we decided to group patients into NYHA I-II versus NYHA III-IV. Moreover, both Kyn and NT-proBNP were significantly higher in nonsurvivors as compared with survivors (Fig. 2A).

Table 2 shows the results of the logistic regression analysis with death as the dependent variable. We identified the following significant univariate predictors: plasma Kyn, LVEF, 


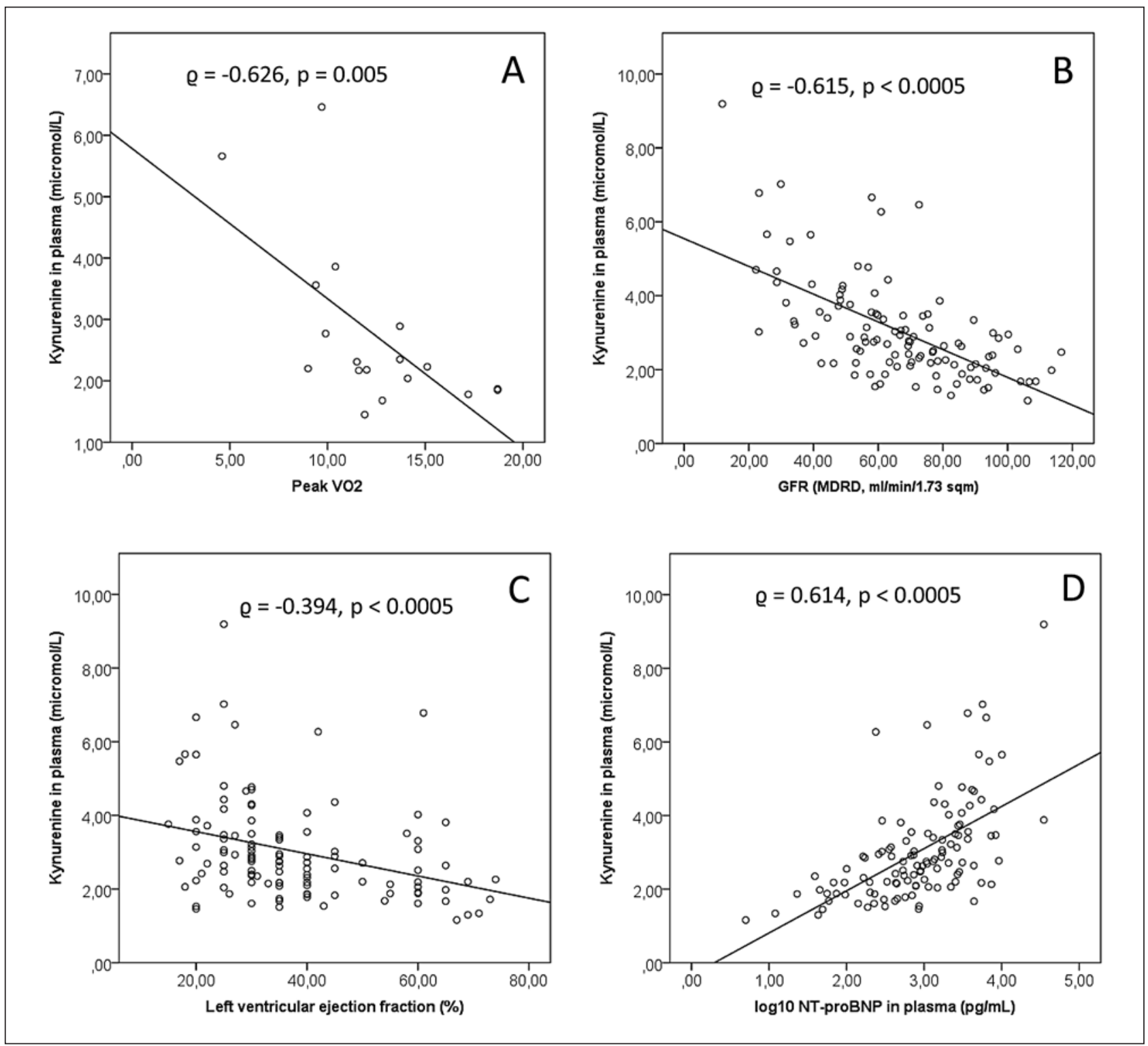

Fig. 3. Correlations of Kyn in the CHF cohort. Kyn correlates negatively with peak $\mathrm{VO}_{2}$ (A), GFR (MDRD; B), as well as LVEF (C), and positively with NT-proBNP (D).

left ventricular end-diastolic diameter (LVEDD), height, plasma sodium, and presence of CAD. It is noteworthy that NT-proBNP was not predictive upon univariate analysis. Correspondingly, the area under the ROC curve for Kyn (0.70) tended to be higher than that for NT-proBNP (0.63; $p=0.26$; Fig. 2B). When we combined Kyn and NT-proBNP by multivariate binary logistic regression, only Kyn contributed significantly (OR 1.75, 95\% CI 1.132.70); thus, the combined ROC analysis did not result in any improvement (not shown). Regarding the multivariate prediction model shown in Table 2, we chose the following model building: as significant univariate correlations of Kyn with LVEDD ( $\varrho=0.326, p=$ $0.001), \operatorname{LVEF}(\varrho=-0.394, p<0.001)$, and sodium $(\varrho=-0.212, p=0.031)$, but not with height ( $\varrho=-0.023, p=0.809$ ) were found, we constructed Models 1 (Kyn, height) and 2 (Kyn, height, CAD) as preliminary, stepwise multivariate models to exclude any possible collin- 
Table 3. Results of multiple linear regression: determinants of Kyn in the AICD cohort

\begin{tabular}{llll}
\hline Parameter & $\begin{array}{l}\text { Regression } \\
\text { coefficient B }\end{array}$ & $\begin{array}{l}\text { Standard } \\
\text { error }\end{array}$ & Significance \\
\hline GFR (MDRD) & -0.005 & 0.0015 & $p<0.001$ \\
hsCRP & 0.091 & 0.0211 & $p<0.001$ \\
Tryptophan & 0.007 & 0.0014 & $p<0.001$ \\
Age & 0.005 & 0.0023 & $p=0.046$ \\
Zonulin & -0.193 & 0.1092 & $p=0.077$ \\
LVEF & -0.003 & 0.0022 & $p=0.140$ \\
CML-calprotectin & -0.017 & 0.0114 & $p=0.133$ \\
\hline
\end{tabular}

Only determinants with $p<0.20$ are shown. Kyn, kynurenine; hsCRP, high-sensitivity CRP; CML-calprotectin, carboxy-methyl-lysine calprotectin; LVEF, left ventricular ejection fraction.

earity issues. The final Model 3 incorporates all univariate candidates whether or not they correlate with the covariable Kyn. Kyn was still a significant multivariate predictor in Model 1 (Table 2), close to significance in Model 2, but not part of the final model which contained sodium, LVEDD, and height.

Kyn Predicts Severely Compromised Left Ventricular Function

We compared the ability of Kyn or NT-proBNP to discriminate at a threshold of LVEF < $30 \%$ (Fig. 2C). Both single ROC curves had an AUC of 0.74; a combined ROC analysis did not result in improved discrimination which may be attributable to the high degree of correlation between Kyn and NT-proBNP (see below).

Kyn Associates with Established Markers of CHF Severity

Figure 3 summarizes the different univariate associations of Kyn with markers of disease severity: Kyn correlated inversely with peak $\mathrm{VO}_{2}$ (Fig. 3A; determined in 18 of 114 patients in whom exercise testing by spiroergometry had been performed), glomerular filtration fraction (GFR) according to the MDRD formula (Fig. 3B), and LVEF (Fig. 3C). Moreover, there was a strong correlation of Kyn with NT-proBNP (Fig. 3D).

\section{AICD Cohort}

Kyn Associates with Metabolic Inflammation, Kidney Function, and Tryptophan

The following parameters showed significant univariate associations with Kyn and were therefore included in the multiple linear regression analysis: zonulin $(\varrho=-0.265)$, carbonylated proteins $(\varrho=0.295)$, creatinine $(\varrho=0.478)$, hsCRP $(\varrho=0.322)$, tryptophan $(\varrho=0.342)$, carboxy-methyl-lysine calprotectin $(\varrho=-0.112)$, LVEF $(\varrho=-0.215)$, GFR according to MDRD $(\varrho=-0.521)$, age $(\varrho=0.345)$, and severity of CAD $(1-, 2-$, or 3 -vessel disease; $\varrho=0.177)$. In addition, patients with type- 2 diabetes $(n=42$; Table 1$)$ had significantly higher Kyn levels (3.70 $\mu \mathrm{mol} / \mathrm{L}$, range 3.12-4.55) than non-diabetic patients (3.30 $\mu \mathrm{mol} / \mathrm{L}$, range $2.60-4.22)$. Table 3 summarizes the results of the multiple linear regression analysis which demonstrated highly significant associations of Kyn with GFR, hsCRP, and tryptophan, as well as a significant correlation with age. 


\section{Kidney \\ Blood Pressure \\ Research}

\begin{tabular}{l|l}
\hline Kidney Blood Press Res 2019;44:765-776 \\
\hline DOI: 10.1159/000501483 & $\begin{array}{l}\text { @ 2019 The Author(s). Published by S. Karger AG, Basel } \\
\text { www.karger.com/kbr }\end{array}$ \\
\hline
\end{tabular}

Dschietzig et al.: Kynurenine and Heart Failure

\section{Discussion}

This clinical investigation was undertaken in order to substantiate the role of Kyn as a biomarker in CHF. To this end, we gathered data in 2 independent CHF cohorts and arrived at the following main conclusions.

Kyn increased with severity of CHF; for predicting death from any cause it performed better than the established CHF biomarker, NT-proBNP. Kyn exhibited highly significant inverse correlations with peak $\mathrm{VO}_{2}, \mathrm{LVEF}$, and GFR, and it correlated closely with NT-proBNP. In an attempt to identify clinical and biochemical key determinants of Kyn, we found that GFR and hsCRP as well as tryptophan plasma levels had the most significant impact.

The general finding of plasma levels of Kyn being elevated in patients with CHF was first reported by Konishi et al. [13]. The authors found circulating Kyn increased in patients with HFrEF (mean \pm SD $3.5 \pm 1.5 \mu \mathrm{mol} / \mathrm{L}$ ) and HFpEF (3.4 $\pm 1.3 \mu \mathrm{mol} / \mathrm{L}$ ) as compared with control individuals $(2.4 \pm 1.1 \mu \mathrm{mol} / \mathrm{L})$, with most of their CHF patients being in NYHA class II. Our current investigation extends this report by adding data on the course of Kyn levels with NYHA class. Whereas plasma levels in our NYHA I and NYHA II/III patients are comparable to those measured by Konishi et al. [13] in controls and CHF, respectively, circulating Kyn rose as high as 6-8 $\mu \mathrm{mol} / \mathrm{L}$ in our patients in NYHA IV. As already mentioned in the Introduction, the key stimulus to Kyn increase is the well-established upregulation of inflammatory cytokines in CHF [14].

In our logistic regression analysis, Kyn proved to be the only parameter among a number of CHF (NT-proBNP), inflammatory (CRP, zonulin), and oxidative stress markers (nitrotyrosine, oxidized LDL) that showed predictive value for death (Table 2). Kyn was no longer significant in the final multivariate model comprising sodium, height, and LVEDD, which, however, may be attributable to the relatively small event numbers in this pilot study. While low sodium and high LVEDD are established risk predictors in CHF, the appearance of height seems counterintuitive at first sight. Nevertheless, low height has repeatedly been demonstrated to represent an independent risk factor, e.g., for all-cause mortality and hospitalization in heart failure patients with cardiac resynchronisation therapy [15], for early mortality after transplantation [16], as well as for major adverse coronary events in a general population [17].

To date, there is only one additional publication regarding death prediction in CHF by Kyn [18]. The authors determined that out of 42 biologically relevant serum amine-based metabolites, only N-methyl-L-histidine, symmetric dimethylarginine, and L-Kyn predicted ventricular arrhythmia-induced AICD shocks and all-cause mortality in two independent cohorts of AICD carriers with CHF, PROSE-ICD, and GRADE. There is circumstantial evidence in support of this work from findings in rodents demonstrating protection against pancreatitis-related multiple organ failure and death by genetic deficiency or selective pharmacological inhibition of Kyn monooxygenase, an enzyme converting Kyn into 3-hydroxyKyn [19]. Along the same lines, in 245 patients suffering from out-of-hospital cardiac arrest enrolled in the FINNRESUSCI Study [12], metabolites of the Kyn pathway (Kyn, kynurenic acid, 3-hydroxyanthranilic acid) were significantly increased in patients dead versus alive both at the ICU and after 12 months. Multivariable logistic regression showed that all 3 Kyn metabolites impacted significantly on ICU mortality, whereas high kynurenic acid and 3-hydroxyanthranilic acid were revealed as significant predictors of a 12-month poor outcome.

In our previous work [13], we reported on the inverse Kyn-spiroergometric peak $\mathrm{VO}_{2}$ association in CHF patients, which remained significant after adjustment for age, sex, BMI, and hemoglobin. This inverse correlation could be confirmed here, albeit in a much smaller subcohort (Fig. 3). Exercise-induced upregulation of Kyn aminotransferases via PGC-1 $\alpha$ contributes to local Kyn degradation in skeletal muscle [20], which may account for the 


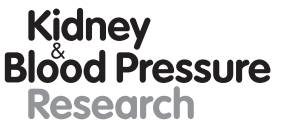

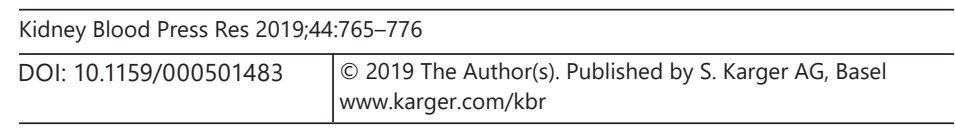

Dschietzig et al.: Kynurenine and Heart Failure

observed relation between plasma Kyn and exercise performance. Besides, this exerciserelated mechanism was shown to protect against depression since kynurenic acid, the local Kyn metabolite produced by skeletal Kyn aminotransferases, cannot permeate the bloodbrain barrier [20]. Another fact that may contribute to the described constellation between Kyn and exercise capacity is tryptophan depletion by IDO upregulation [21]: enhanced tryptophan degradation was shown to inhibit the proliferation of bone marrow cells, which may result in anemia. This holds particularly true of end-stage renal or cardiac disease $[22,23]$ in which low circulating tryptophan levels were found.

In our AICD cohort, we established highly significant associations of Kyn with GFR, hsCRP, and tryptophan, as well as a significant increase with age (Table 3). While the increase with tryptophan obviously reflects the common metabolic pathway [1], the inverse relation to renal function is certainly attributable to chronic immune upregulation/dysregulation in renal patients. Thus, plasma IDO levels and activity were reported to be increased with the severity of renal disease [24, 25]. IDO levels, in turn, correlated well with T cell depletion in renal disease [25], which established a link to immunosuppression. Vice versa, we have recently demonstrated baseline Kyn prior to coronary angiography to reflect the propensity to develop contrast-induced nephropathy [26], which renders Kyn an appropriate risk marker for cardiorenal syndrome.

Eventually, despite significant univariate (inverse) correlations of Kyn with LVEF in both cohorts, LVEF was no longer a significant determinant of Kyn in multiple regression analysis (Table 3). This fact - together with hsCRP appearing among the major determinants in that analysis - most likely points to the fact that Kyn is primarily an inflammation-driven, not a remodeling-driven, parameter.

As to the limitations of our study, we should mention that both study cohorts were of modest size with low absolute numbers of events (deaths) and a rather short follow-up period in the CHF cohort, which necessitates larger studies to exclude chance findings. Also, we had no time-to-event data owing to logistical limitations which rendered Cox analysis impossible.

\section{Conclusion}

This work reinforces the concept of Kyn being a new and valuable biomarker of CHF, with particular attention placed on its ability to predict mortality and reflect exercise capacity.

\section{Acknowledgements}

We would like to acknowledge the expert technical and logistic assistance from Dr. B. Hafen and Mr. A. Böcher (Immundiagnostik AG).

\section{Statement of Ethics}

All patients in the cohorts described provided informed written consent to have data from their medical records used in research. All data were fully anonymized before being accessed by researchers. This study was approved by the Ethics Committee of the Charité University Medicine Berlin (approval No. EA1/004/13) and by the Ethics Committee of the Justus Liebig University of Giessen (Kerckhoff Clinic Bad Nauheim; BioData Bank 144/11) and has been performed in accordance with the ethical standards laid down in the 1964 Declaration of Helsinki and its later amendments. 


\section{Kidney \\ Blood Pressure \\ Research}

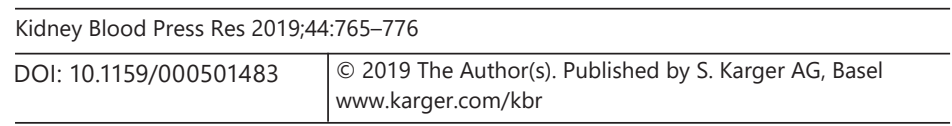

\section{Disclosure Statement}

The authors declare the following conflicts of interest. T.B.D. is an employee of Immundiagnostik. F.P.A. holds the majority of shares in Immundiagnostik. K.-H.K. is CEO and owner of Neuroimmun $\mathrm{GmbH}$, the manufacturer of the kynurenine kit which is distributed by Immundiagnostik. K.-H.K. has applied or owns patents on "immunoassays for biogenic amines” (PCT/EP2011/069936, EP20110797208, EP2612147A1, W02012065912A1). V.M. is an employee of the Kerckhoff Clinic GmbH, Bad Nauheim.

\section{Funding Sources}

This study was funded by Immundiagnostik AG and Neuroimmun GmbH. As the first author (T.B.D.) and further co-authors (J.R., F.P.A.) are either employees (T.B.D., J.R.) or shareholders (F.P.A.) of Immundiagnostik AG, with another co-author (K.-H.K.) being the CEO of Neuroimmun $\mathrm{GmbH}$, the Role of Funders in this work was not limited to mere financial support, but included a role in the study design, data collection and analysis, decision to publish, and preparation of the manuscript as summarized in the Author Contributions statement.

\section{Author Contributions}

Conceptualization: Thomas Bernd Dschietzig, Christoph Melzer. Data curation: Katrin Sasse, Felix Boschann, Robert Klüsener, Jana Ruppert. Formal analysis: Thomas Bernd Dschietzig, Dragic Bankovic, Veselin Mitrovic. Investigation: Karl-Heinz Kellner, Katrin Sasse, Felix Boschann, Robert Klüsener, Jana Ruppert, Franz Paul Armbruster, Andreas Meinitzer. Methodology: Karl-Heinz Kellner, Jana Ruppert, Franz Paul Armbruster, Andreas Meinitzer. Project administration: Thomas Bernd Dschietzig, Christoph Melzer, Veselin Mitrovic. Resources: Thomas Bernd Dschietzig, Christoph Melzer, Franz Paul Armbruster, Veselin Mitrovic. Supervision: Thomas Bernd Dschietzig, Christoph Melzer, Veselin Mitrovic. Writing - original draft preparation: Thomas Bernd Dschietzig, Katrin Sasse, Felix Boschann, Robert Klüsener. Writing - review and editing: Andreas Meinitzer, Christoph Melzer, Veselin Mitrovic, KarlHeinz Kellner, Franz Paul Armbruster, Dragic Bankovic, Jana Ruppert.

\section{References}

1 Hassanain HH, Chon SY, Gupta SL. Differential regulation of human indoleamine 2,3-dioxygenase gene expression by interferons-gamma and -alpha. Analysis of the regulatory region of the gene and identification of an interferon-gamma-inducible DNA-binding factor. J Biol Chem. 1993 Mar;268(7):5077-84.

2 Richard DM, Dawes MA, Mathias CW, Acheson A, Hill-Kapturczak N, Dougherty DM. L-Tryptophan: basic metabolic functions, behavioral research and therapeutic indications. Int J Tryptophan Res. 2009 Mar;2:45-60.

3 Babcock TA, Carlin JM. Transcriptional activation of indoleamine dioxygenase by interleukin 1 and tumor necrosis factor alpha in interferon-treated epithelial cells. Cytokine. 2000 Jun;12(6):588-94.

4 Fujigaki S, Saito K, Sekikawa K, Tone S, Takikawa O, Fujii H, et al. Lipopolysaccharide induction of indoleamine 2,3-dioxygenase is mediated dominantly by an IFN-gamma-independent mechanism. Eur J Immunol. 2001 Aug;31(8):2313-8.

5 Schwarcz R, Bruno JP, Muchowski PJ, Wu HQ. Kynurenines in the mammalian brain: when physiology meets pathology. Nat Rev Neurosci. 2012 Jul;13(7):465-77.

6 Katz JB, Muller AJ, Prendergast GC. Indoleamine 2,3-dioxygenase in T-cell tolerance and tumoral immune escape. Immunol Rev. 2008 Apr;222(1):206-21.

7 Fujigaki H, Saito K, Lin F, Fujigaki S, Takahashi K, Martin BM, et al. Nitration and inactivation of IDO by peroxynitrite. J Immunol. 2006 Jan;176(1):372-9. 
Dschietzig et al.: Kynurenine and Heart Failure

8 Wang Y, Liu H, McKenzie G, Witting PK, Stasch JP, Hahn M, et al. Kynurenine is an endothelium-derived relaxing factor produced during inflammation. Nat Med. 2010 Mar;16(3):279-85.

9 Pawlak K, Domaniewski T, Mysliwiec M, Pawlak D. The kynurenines are associated with oxidative stress, inflammation and the prevalence of cardiovascular disease in patients with end-stage renal disease. Atherosclerosis. 2009 May;204(1):309-14.

10 Pedersen ER, Svingen GF, Schartum-Hansen H, Ueland PM, Ebbing M, Nordrehaug JE, et al. Urinary excretion of kynurenine and tryptophan, cardiovascular events, and mortality after elective coronary angiography. Eur Heart J. 2013 Sep;34(34):2689-96.

11 Pedersen ER, Tuseth N, Eussen SJ, Ueland PM, Strand E, Svingen GF, et al. Associations of plasma kynurenines with risk of acute myocardial infarction in patients with stable angina pectoris. Arterioscler Thromb Vasc Biol. 2015 Feb;35(2):455-62.

12 Ristagno G, Latini R, Vaahersalo J, Masson S, Kurola J, Varpula T, et al.; FINNRESUSCI Investigators. Early activation of the kynurenine pathway predicts early death and long-term outcome in patients resuscitated from out-of-hospital cardiac arrest. J Am Heart Assoc. 2014 Aug;3(4):e001094.

13 Konishi M, Ebner N, Springer J, Schefold JC, Doehner W, Dschietzig TB, et al. Impact of Plasma Kynurenine Level on Functional Capacity and Outcome in Heart Failure?- Results From Studies Investigating Co-morbidities Aggravating Heart Failure (SICA-HF). Circ J. 2016 Dec;81(1):52-61.

14 Anker SD, Egerer KR, Volk HD, Kox WJ, Poole-Wilson PA, Coats AJ. Elevated soluble CD14 receptors and altered cytokines in chronic heart failure. Am J Cardiol. 1997 May;79(10):1426-30.

15 Linde C, Cleland JG, Gold MR, Claude Daubert J, Tang AS, Young JB, et al. The interaction of sex, height, and QRS duration on the effects of cardiac resynchronization therapy on morbidity and mortality: an individual-patient data meta-analysis. Eur J Heart Fail. 2018 Apr;20(4):780-91.

16 Pozo-Laderas JC, Rodriguez-Peralvarez M, Munoz-Villanueva MC, Rivera-Espinar F, Durban-Garcia I, MunozTrujillo J, et al. Pretransplant predictors of early mortality in adult recipients of liver transplantation in the MELD-Na Era. Med Intensiva. 2019;43(5):261-9.

17 Park CS, Choi EK, Han KD, Lee HJ, Rhee TM, Lee SR, et al. Association between adult height, myocardial infarction, heart failure, stroke and death: a Korean nationwide population-based study. Int J Epidemiol. 2018 Feb;47(1):289-98.

18 Zhang Y, Blasco-Colmenares E, Harms AC, London B, Halder I, Singh M, et al. Serum amine-based metabolites and their association with outcomes in primary prevention implantable cardioverter-defibrillator patients. Europace. 2016 Sep;18(9):1383-90.

19 Mole DJ, Webster SP, Uings I, Zheng X, Binnie M, Wilson K, et al. Kynurenine-3-monooxygenase inhibition prevents multiple organ failure in rodent models of acute pancreatitis. Nat Med. 2016 Feb;22(2):202-9.

20 Agudelo LZ, Femenía T, Orhan F, Porsmyr-Palmertz M, Goiny M, Martinez-Redondo V, et al. Skeletal muscle PGC-1 $\alpha 1$ modulates kynurenine metabolism and mediates resilience to stress-induced depression. Cell. 2014 Sep;159(1):33-45.

21 Schroecksnadel K, Wirleitner B, Fuchs D. Anemia and congestive heart failure. Circulation. 2003 Aug; 108(6):e41-2.

22 Debnath S, Velagapudi C, Redus L, Thameem F, Kasinath B, Hura CE, et al. Tryptophan metabolism in patients with chronic kidney disease secondary to type 2 diabetes: relationship to inflammatory markers. Int J Tryptophan Res. 2017 Mar;10:1178646917694600.

23 Xu Z, Zhang L, Li J, Zhao Y, Chen X. Metabonomic Profiling Reveals Difference in Altered Metabolic Pathways Between Chronic Kidney Disease and High-Fat-Induced Insulin Resistance in Rats. Kidney Blood Press Res. 2018;43(4):1199-211.

24 Bao YS, Ji Y, Zhao SL, Ma LL, Xie RJ, Na SP. Serum levels and activity of indoleamine2,3-dioxygenase and tryptophanyl-tRNA synthetase and their association with disease severity in patients with chronic kidney disease. Biomarkers. 2013 Aug;18(5):379-85.

25 Eleftheriadis T, Yiannaki E, Antoniadi G, Liakopoulos V, Pissas G, Galaktidou G, et al. Plasma indoleamine 2,3-dioxygenase and arginase type I may contribute to decreased blood T-cell count in hemodialysis patients. Ren Fail. 2012;34(9):1118-22.

26 Reichetzeder C, Heunisch F, Einem GV, Tsuprykov O, Kellner KH, Dschietzig T, et al. Pre-Interventional Kynurenine Predicts Medium-Term Outcome after Contrast Media Exposure Due to Coronary Angiography. Kidney Blood Press Res. 2017;42(2):244-56. 